\title{
A palpable tender abdominal mass in a patient with endocarditis on antibiotics for 5 weeks
}

\author{
Stephanie L. Smeltzer ${ }^{1^{*}}$, Lourdes C. Corman ${ }^{2}$ \\ ${ }^{1}$ Huntsville Regional Campus, University of Alabama at Birmingham School of Medicine, Huntsville, USA; \\ *Corresponding Author: shlarson@uab.edu \\ ${ }^{2}$ Department of Medicine, Huntsville Regional Campus, University of Alabama at Birmingham School of Medicine, Huntsville, USA
}

Received 25 January 2013; revised 27 February 2013; accepted 8 March 2013

\begin{abstract}
Mycotic aneurysms are a rare finding in the post-antibiotic era but must still be considered as a cause of abdominal pain in a patient with endocarditis. We present a case of a 47-year-old man with a history of IV drug use and a prior episode of endocarditis who developed a mycotic aneurysm while hospitalized on IV antibiotic therapy for aortic valve endocarditis. Due to their life-threatening character and often lack of evident clinical picture, mycotic aneurysms should still remain in high suspicion for a patient with abdominal pain in the setting of endocarditis.
\end{abstract}

Keywords: Mycotic Aneurysm; Pseudoaneurysm; Enterococcal Endocarditis

\section{INTRODUCTION}

Mycotic aneurysms are a potentially life-threatening and unusual presentation of bacterial endocarditis. Emboli are released from the heart that lodge into the vaso vasorum of an arterial wall, and an inflammatory reaction is then set up. The arterial wall is weakened, most likely from hypersensitivity, leading to an aneurysm [1]. The superior mesenteric artery is an uncommon location for aneurysms, as only $5.5 \%$ of visceral aneurysms are found in this location. Nevertheless, $50 \%$ of aneurysms of the superior mesenteric artery (SMA) are known to be mycotic in origin [2]. Although they are uncommon, $38 \%-50 \%$ of all visceral aneurysms will rupture, with often fatal consequences [3]. Therefore, it is paramount that such aneurysms be diagnosed in their early stage before such disastrous consequences can occur.

\section{CASE REPORT}

A 47-year-old, known IV drug user presented to the emergency department with abdominal pain, weight loss and non-bloody emesis for the past 4 weeks. He denied fever. The murmur of aortic regurgitation was present, and $\mathrm{CT}$ of the abdomen revealed mesenteric panniculitis (Figure 1). Blood cultures grew Enterococcus faecalis. An echocardiogram revealed a large vegetation on the ventricular side of the aortic valve. He was started on IV vancomycin, and his abdominal discomfort improved.

On week 5 , the abdominal pain returned. The vital signs were stable. He had moderate abdominal tenderness without peritoneal signs, and a $6 \mathrm{~cm}$ diameter periumbilical tender pulsating mass was noted. The mass was thought to be transmitting aortic pulsations. Previous hemoccult testing was positive for blood.

A repeat abdominal CT was performed which showed a soft tissue mass within the retroperitoneum and mesentery which was quickly growing and suggestive of a lymphoma (Figure 2). Instead, a pseudoaneurysm of the superior mesenteric artery was found. Embolization of the pseudoaneurysm was performed with 37 coils. His abdominal pain improved after the procedure. He completed the 6 week course of IV antibiotics and was discharged home a week later after declining any surgical intervenetion.

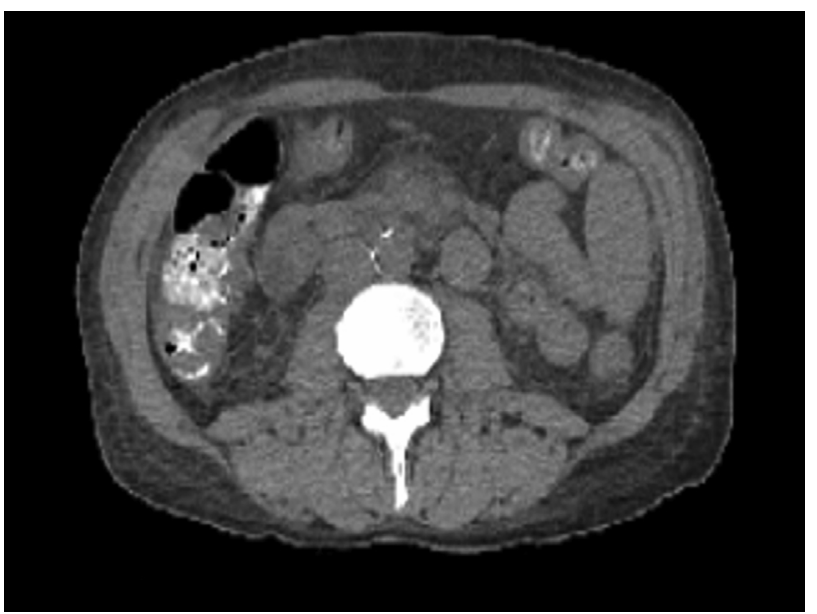

Figure 1. CAT of abdomen and pelvis upon admission showing prominent soft tissue in the root of the mesentery associated with the central mesenteric vessels most characteristic of mesenteric panniculitis. 


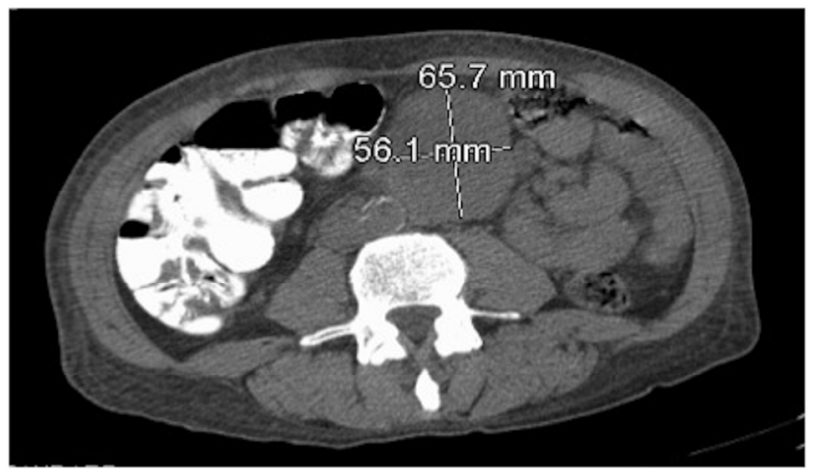

Figure 2. CAT of abdomen and pelvis showing a soft tissue mass which appears to be rapidly growing.

\section{DISCUSSION}

In a patient with abdominal pain, nausea and vomiting, gastrointestinal bleeding, and a history of endocarditis, mycotic aneurysm should be considered. Although quite rare, due to recent advances in technology, the incidence has risen in recent decades. Mycotic aneurysms can be most commonly found in the hepatic artery, splenic artery, descending aorta, intracranial arteries, and the renal artery [1]. Septic emboli are lodged into the vaso vasorum of a vessel wall, with subsequent spread of the infection and weakening of the vessel wall. The presenting complaint of abdominal pain is usually as a result of either mesenteric ischemia or direct aneurysm expansion. Often, mycotic aneurysms are asymptomatic [4].

If suspected, diagnostic testing with Doppler ultrasound, CAT scan, or MRI of the abdomen and pelvis should then be performed. If aneurysm is found on imaging, appropriate treatment involves either surgical resection or endovascular therapy. Endovascular therapy with coil embolization or stent-graft therapy is usually reserved for patients not hemodynamically stable for surgery. Once the aneurysm has already ruptured, either emergency laporotomy with aneurysmectomy or embolization with metallic coils is recommended. The survival rate after rupture is around $40 \%$ [2]. In a patient with comorbidies or after failure of primary treament, endovascular therapy using stent or endovascular coils is preferred [5].

In this case, a leak was already present in the superior mesenteric artery, and the hematoma was contained by surrounding tissues. Embolization allowed the growing pseudoaneurysm to be occluded before the hemodynamic stability collapsed. Follow-up at regular intervals can be arranged if recurrence is suspected.

\section{REFERENCES}

[1] Christophe, C., et al. (1985) Ruptured mycotic aneurysm of the superior mesenteric artery secondary to bacterial endocarditis in a 6-year-old girl. Pediatric Radiology, 15, 202-204. doi:10.1007/BF02388614

[2] Akkary, E., Cramer, T., Patel, M., Kanthimathinathan, V. and Phan, T. (2010) Superior mesenteric artery aneurysm: An uncommon disease with potentially serious complications. West Virginia Medical Journal, 106, 10-14.

[3] Stone, W., Abbas, M., Cherry, K., Fowl, R. and Gloviczki, P. (2002) Superior mesenteric artery aneurysms: Is presence an indication for intervention? Journal of Vascular Surgery, 36, 234-237. doi:10.1067/mva.2002.125027

[4] Lorelli, D., Cambria, R., Seabrook, G. and Towne, J. (2003) Diagnosis and management of aneurysms involving the superior mesenteric artery and its braches: A report of four cases. Vascular and Endovascular Surgery, 37, 59-66. doi:10.1177/153857440303700108

[5] Sachdev, U., et al. (2006) Management of aneurysms involving branches of the celiac and superior mesenteric arteries: A comparison of surgical and endovascular therapy. Journal of Vascular Surgery, 44, 718-724. doi:10.1016/j.jvs.2006.06.027 